\title{
Semantically factoid question answering using fuzzy SVM named entity recognition
}

\begin{abstract}
Named Entity Recognition (NER) and Question Answering (QA) are fundamental tasks and they are the cores of natural language processing (NLP) system. NER, a sub problem of Information Extraction (IE), involves recognizing and extracting name entities like Persons, Locations, Organizations, Dates and Times from electronics resources and text. Question Answering (QA) is a type of Information Retrieval (IR), attempts to deal with a wide range of question. In this paper we propose a semantically Factoid Question Answering model using Fuzzy Support Vector Machine Named Entity Recognizer component called FSVM. In this model we applied the FSVM NE recognizer to filter Question Answering system results have token by IR and return exact expect result to the user. This paper shows how the Fuzzy NER can applied in information retrieval (IR) systems in applications like Question Answering (QA). We show a model to improve precision in QA by semantically NER and reducing Answer Finder input data.
\end{abstract}

